receptor, markedly increased intestinal inflammation and neutrophil influx was seen in models of acute colitis.

"Overall, we conclude that constitutive secretion of the NAE class of endocannabinoids via the P-gp efflux pump acts to dampen inflammation at the intestinal mucosal surface, thus working as a brake to oppose $\mathrm{HxA}_{3}$-mediated inflammation," concludes McCormick. "This work opens up potentially exciting areas of study on the neurobiological functions of the endocannabinoid pathway and the importance of the brain-gut axis."

These new findings give insight into pathways crucial for maintaining epithelial homeostasis that might occur at other mucosal surfaces in the body. The investigators are also hopeful that their work could lead to targeted local therapies for IBD that currently rely on systemic immunosuppressive agents with potential adverse effects.

Iain Dickson

ORIGINAL ARTICLE Szabady, R. L. et al. Intestinal P-glycoprotein exports endocannabinoids to prevent inflammation and maintain homeostasis. J.Clin. Invest. https://doi.org/10.1172/JCI96817 (2018)

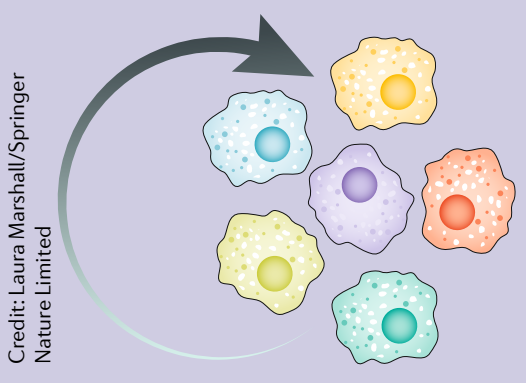

however, inhibition of monocyte recruitment in this gMac depletion model led to abnormal intestinal physiology and function, including lengthened intestinal transit times and vascular leakage. "This study revisits the current paradigm of gMac ontogeny and heterogeneity and highlights the importance of evaluating the role of distinct gMac subsets in gastrointestinal function," write the authors. They suggest that future studies should explore the role of gMac subsets in disease models and human biopsy tissue.

Hugh Thomas

ORIGINAL ARTICLE De Schepper, S. et al. Self-maintaining gut macrophages are essential for intestinal homeostasis. Cell 175, 400-415.e13 (2018)

\title{
Personalized response prediction
}

Results from the phase II trial PROSPECT-C in patients with metastatic colorectal cancer treated with cetuximab show that monitoring mutations in circulating free DNA (cfDNA) with frequent liquid biopsies enables individualized prediction of treatment response. The researchers also found that $\sim 50 \%$ of patients had mutations in RAS pathways at baseline and did not respond to treatment, although they had been classified as RAS wild-type following previous genetic testing.

" $40-50 \%$ of patients receiving cetuximab have some benefit, but many patients relapse after an initial response and become resistant to the drug," explains Nicola Valeri, senior author of the study. "We know that resistance to cetuximab is largely due to the tumour evolving and adapting to therapy through the acquisition of mutations in the RAS pathway. We used blood samples taken every 4 weeks (quite frequently for this type of study) to monitor cancer evolution during treatment and see whether there were some measurements that predicted whether and when patients would relapse."

Overall, following a median 10.7 months of single-agent cetuximab treatment, $20.0 \%, 24.4 \%$ and $46.7 \%$ of patients experienced partial response, stable disease, and progressive disease, respectively. Median progression-free survival was 2.6 months $(95 \% \mathrm{Cl} 1.9-4.2)$ and overall survival was 8.2 months ( $95 \% \mathrm{Cl} 4.2-12.0$ ).

For the analysis of cfDNA mutation status in blood samples, patients were grouped into two cohorts: in an initial set of 22 patients (cohort 1), cfDNA was analysed by digital-droplet PCR to assess specific mutation hotspots; in the second cohort of 17 patients, cfDNA was analysed by next-generation sequencing of multiple genes. Investigation of mutations in the RAS pathway in cfDNA taken at baseline showed that $~ 50 \%$ of patients had aberrations before commencing cetuximab treatment, which included mutations in KRAS, NRAS, BRAF, PIK3C, EGFR and FGFR, as well as ERBB2 amplification. Presence of such mutations was associated with reduced progression-free and overall survival and these patients had a trend towards poor response rates compared with those with wild-type genotypes ( $0 \%$ versus $36.4 \%$; $\mathrm{P}=0.09$ ). Concentration of cfDNA did not differ between patients with or without mutations. Analysis of blood samples taken at disease progression showed that $86.3 \%$ of those in cohort 1 had RAS pathway mutations at that time point. In $75 \%$ of those who had a partial response, aberrations were detectable in cfDNA before progression was clinically or radiologically detected. In both patients with primary resistance and those with acquired resistance, multiple independent RAS-mutant subclones were identified.

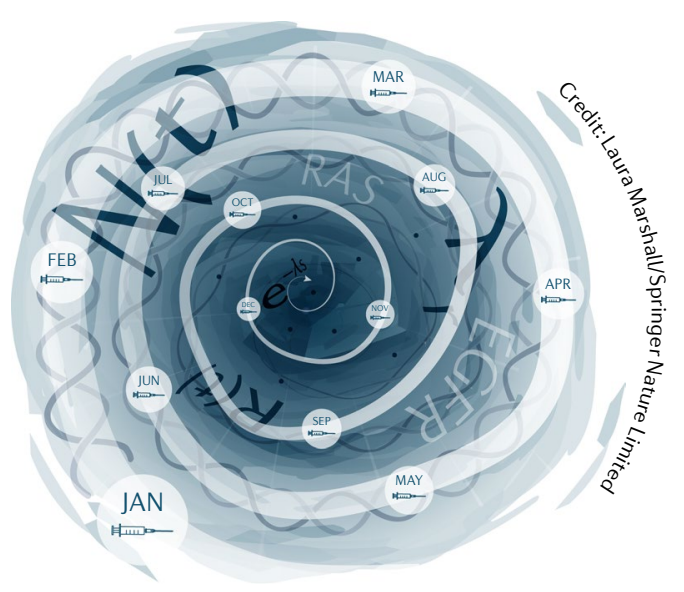

The team then compared cfDNA analysis results with sequencing data from metastases samples taken at relevant time points. Of 24 mutations detected in cfDNA from 14 patients, $79 \%$ of mutations were observed in $\geq 1$ paired biopsy sample. In 6 patients who also had archival tissue available, several mutations were present in both archival and pretreatment biopsy samples; however, allele frequency of mutations was below the detection threshold of clinically approved assays, explaining the initial RAS-wild-type classification of patients who were then found to have mutations in this trial.

Finally, the researchers developed a mathematical model to predict time to progression on the basis of cfDNA mutations and carcinoembryonic antigen levels (CEA; a marker of tumour burden) measured in the blood samples. The resultant model was able to measure patient-specific resistance dynamics and give a personalized prediction of time to progression, and highlighted the importance of blood sampling at least every 4 weeks.

"This study demonstrates that it is possible, using the right monitoring approach, to make quantitative predictions about the future course of cancer in individual patients," summarizes Andrea Sottoriva, study co-lead. "Our results need to be confirmed in larger trials, but our study opens the door for novel research on cancer forecasting, as well as new types of clinical trials in which frequent liquid biopsies are used to closely monitor patients, prompting change of treatment, combination of treatments or scheduling of drug holidays to re-sensitize cancer populations, when required."

Clemens Thoma

ORIGINAL ARTICLE Khan, K. H. et al. Longitudinal liquid biopsy and mathematical modeling of clonal evolution forecast time to treatment failure in the PROSPECT-C phase Il colorectal cancer clinical trial. Cancer Discov. https://doi.org/10.1158/2159-8290.CD17-0891 (2018) 